\title{
Celastrol attenuates the remodeling of pulmonary vascular and right ventricular in monocrotaline-induced pulmonary arterial hypertension in rats
}

\author{
Huayang Li $^{1,2 \#}$, Quan Liu ${ }^{1 \#}$, Yuan Yue ${ }^{1 \# \wedge}$, Shunjun Wang ${ }^{1 \wedge}$, Suiqing Huang ${ }^{1 \wedge}$, Lin Huang ${ }^{1 \wedge}$, Li Luo $^{1 \wedge}$, \\ Yitao Zhang ${ }^{3 \wedge}$, Zhongkai $\mathrm{Wu}^{1,2 \wedge}$ \\ ${ }^{1}$ Department of Cardiac Surgery, The First Affiliated Hospital of Sun Yat-sen University, Guangzhou, China; ${ }^{2}$ NHC Key Laboratory of Assisted \\ Circulation (Sun Yat-sen University), Guangzhou, China; ${ }^{3}$ Department of Cardiovascular, The Sixth Affiliated Hospital of Sun Yat-sen University, \\ Guangzhou, China \\ Contributions: (I) Conception and design: H Li, Z Wu; (II) Administrative support: Z Wu, Y Zhang; (III) Provision of study materials or patients: \\ H Li, Q Liu, Y Yue; (IV) Collection and assembly of data: H Li, Q Liu, Y Yue; (V) Data analysis and interpretation: S Wang, S Huang, L Huang, \\ L Luo; (VI) Manuscript writing: All authors; (VII) Final approval of manuscript: All authors. \\ \#These authors contributed equally to this work. \\ Correspondence to: Prof. Zhongkai Wu. Department of Cardiac Surgery, The First Affiliated Hospital of Sun Yat-sen University, 58 Zhongshan II \\ Road, Guangzhou 510080, China. Email: wuzhk@mail.sysu.edu.cn; Dr. Yitao Zhang. Department of Cardiovascular, The Sixth Affiliated Hospital of \\ Sun Yat-sen University, Guangzhou, China. Email: zhangyt73@mail.sysu.edu.cn.
}

Background: Pulmonary arterial hypertension is a progressive angio-proliferative disease associated with high morbidity and mortality rates. Although the histopathology of pulmonary arterial hypertension is well described, its therapeutic option remains unsatisfactory. This study investigated the effect of celastrol treatment on right ventricular dysfunction, remodeling, and pulmonary vascular remodeling in pulmonary arterial hypertension rats as well as its possible mechanisms.

Methods: Pulmonary arterial hypertension was induced in male Sprague-Dawley rats by a single subcutaneously injection of monocrotaline. After daily delivery of celastrol $(1 \mathrm{mg} / \mathrm{kg})$ or vehicle via intraperitoneal injection for 4 weeks, the effects of celastrol on right ventricular function, fibrosis, and pulmonary vascular remodeling were assessed. The infiltration of macrophages, the expression of inflammatory cytokines, including MCP-1, IL-1 $\beta$, IL-6, and IL-10, and the expression of NF- $\kappa$ B signaling pathway-associated proteins, I $\mathrm{B} \alpha, \mathrm{p}-\mathrm{IKK} \alpha / \beta$ and $\mathrm{p} 65$ were further detected. Finally, the effect of celastrol on human pulmonary artery smooth cells proliferation under hypoxia was studied in vitro.

Results: Rats with pulmonary arterial hypertension had decreased right ventricular function, increased right ventricular fibrosis and pulmonary arteries with interstitial thickening and prominent media hypertrophy. Treatment with celastrol improved right ventricular function, attenuated right ventricular fibrosis and pulmonary vascular remodeling. Significantly decreased macrophage infiltration, reduced levels of pro-inflammatory cytokines, increased level of anti-inflammatory cytokine and inhibited NF- $\mathrm{B}$ signaling pathway were observed in the lung tissues of rats treated with celastrol. Moreover, celastrol significantly suppressed the proliferation of human pulmonary artery smooth cells under hypoxia.

Conclusions: We showed that in rats with pulmonary arterial hypertension, celastrol could improve right ventricular function, attenuate right ventricular and pulmonary vascular remodeling, and inhibit human pulmonary artery smooth cells proliferation under hypoxia. Suppression of the nuclear factor- $\kappa \mathrm{B}(\mathrm{NF}-\kappa \mathrm{B})$ signaling pathway may be a part of the protective mechanism.

^ ORCID: Huayang Li, 0000-0003-4898-2501; Quan Liu, 0000-0003-2585-0960; Yuan Yue, 0000-0002-9304-9078; Shunjun Wang, 00000002-8473-7311; Suiqing Huang, 0000-0001-5496-8867; Lin Huang, 0000-0002-5496-4636; Li Luo, 0000-0001-7809-6821; Yitao Zhang, 0000-0002-7505-5191; Zhongkai Wu, 0000-0003-1184-1182. 
Keywords: Pulmonary arterial hypertension (PAH); celastrol; pulmonary vascular remodeling; right ventricular; nuclear factor- $\kappa \mathrm{B}(\mathrm{NF}-\kappa \mathrm{B})$

Submitted Jun 07, 2021. Accepted for publication Nov 03, 2021.

doi: $10.21037 / \mathrm{cdt}-21-360$

View this article at: https://dx.doi.org/10.21037/cdt-21-360

\section{Introduction}

Pulmonary arterial hypertension (PAH) is characterized by progressive pulmonary arterial remodeling. Arteriopathy in the small to medium-sized distal pulmonary arteries is associated with arterial muscularization, concentric intimal thickening, and plexiform lesion formation, which results in increased pulmonary vascular resistance and leads to right ventricle (RV) overload (1). Although PAH is a rare disease, the incidence of $\mathrm{PAH}$ still ranges from 2.0 to 7.6 cases per million adults per year, and its prevalence varies from 11 to 26 cases per million adults (2). Initially, the RV adapts to the increased afterload by hypertrophying and increasing contractility, but as the disease progresses, the RV fails. Therefore, RV failure is the main cause of death in patients with PAH (3). Despite improvements in treatment options, the overall survival of PAH remains unsatisfactory (4). During the past two decades, several effective medications have been discovered, including prostacyclin analogues and derivatives, endothelin receptor antagonists, phosphodiesterase type 5 (PDE5) inhibitors, and soluble guanylate cyclase (sGC) stimulators (5). Nevertheless, they are primarily vasodilators and none of them target the adverse vascular remodeling and the decline of RV function. Thus, PAH patients continue to face poor health status, frequent hospitalizations, and high risks of premature mortality.

Even though the pathogenesis of PAH is largely unknown, it is increasing being recognized that early and persistent inflammation plays an indispensable role in the development and progression of PAH. Inflammatory cells, such as macrophages, T cells and B cells, infiltrate the plexiform lesions in patients with advanced PAH (6). Moreover, the levels of several inflammatory cytokines and cytokine receptors are changed in PAH lungs, and some of them are correlated with the disease severity and prognosis (7). Among the proinflammatory signaling pathways, nuclear factor- $\kappa \mathrm{B}$ (NF- $\kappa \mathrm{B})$ plays an important role $(8,9)$.

Celastrol, isolated from the root extracts of Tripterygium wilfordii (thunder god vine), is a quinone methide triterpene (10). Increasing evidence suggests that celastrol exerts powerful effects on anti-inflammatory and antioxidant activities $(11,12)$. Indeed, celastrol has been shown to have a protective effect against rheumatoid arthritis, chronic inflammation, and cerebral ischemia injury (13-15). Hu et al. (16) found that celastrol alleviated acute liver inflammation by inhibiting the NF- $\mathrm{\kappa B}$ signaling pathway. Furthermore, recently published reports have demonstrated that celastrol could reduce the cardiac fibrosis that results from transverse aortic constriction (TAC) and angiotensin II in mice $(17,18)$.

Considering the critical role of inflammation in $\mathrm{PAH}$ and the anti-inflammatory effects of celastrol, we therefore set out to examine whether celastrol has protective effects on monocrotaline (MCT)-induced PAH in rats. We present the following article in accordance with the ARRIVE reporting checklist (available at https://cdt. amegroups.com/article/view/10.21037/cdt-21-360/rc).

\section{Methods}

\section{Animals and in vivo treatment}

To limit potential gender differences, we selected only male rats. Young male Sprague-Dawley rats (6-8 weeks, $220-250$ g, purchased from Laboratory Animal Center of Sun Yat-sen University, Guangzhou, China) were housed in a specific pathogen free room with $12 \mathrm{~h}$ light/dark cycle and stable humidity and room temperature $\left(40-70 \%\right.$ and $20-25^{\circ} \mathrm{C}$, respectively). 3 or 4 rats were housed in each cage. Sterile water and rat chow were provided ad libitum. The rats studied 4 weeks after a single subcutaneous injection of MCT (60 mg/kg; Sigma, St. Louis, MO, USA) or equal volume of saline (the Normal group). From the first day, each rat was tagged with an ear tag to identify it, and the MCT-injected rats were randomly divided into two groups. The rats from one group received daily intraperitoneal injection of celastrol $(1 \mathrm{mg} / \mathrm{kg} /$ day, TargetMol, Shanghai, China) for 4 weeks, and an equal volume of vehicle $[0.9 \%$ dimethylsulfoxide $+2 \%$ Tween $80+97.1 \%$ saline] was administered to another group. Therefore, there were three groups and 35 rats: the Normal 


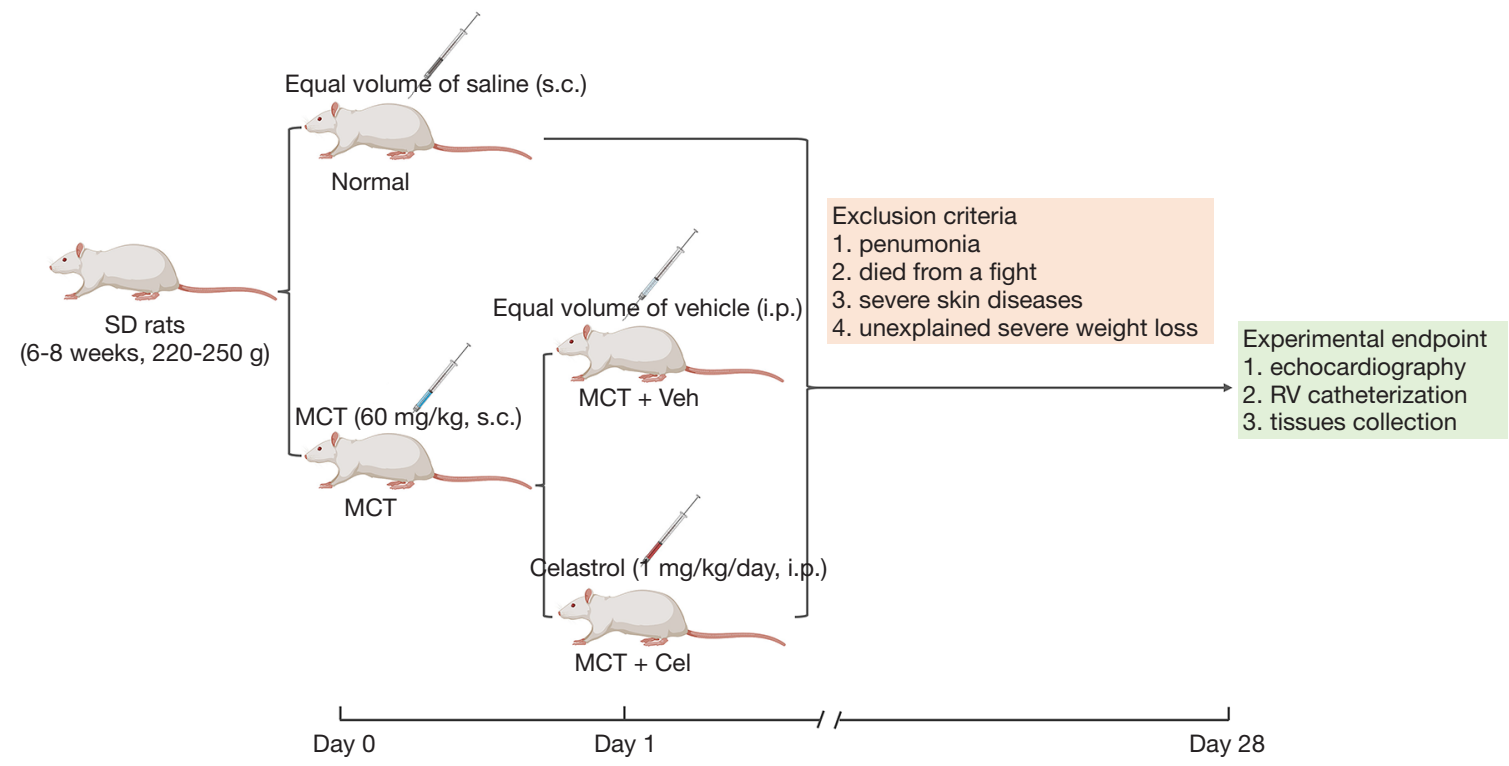

Figure 1 Study design. Sprague-Dawley rats (6-8 weeks, 220-250 g) were subcutaneously injected MCT (60 mg/kg) or equal volume of saline at day 0 . MCT-injected rats were randomly divided into the MCT + Veh and MCT + Cel groups at the day 1 . The rats from MCT + Cel group received daily intraperitoneal injection of celastrol $(1 \mathrm{mg} / \mathrm{kg})$ for 4 weeks, and an equal volume of vehicle was administered to the MCT + Veh group. The four exclusion criteria were illustrated in the figure. MCT, monocrotaline.

group ( $\mathrm{n}=10)$, the MCT + Vel $(\mathrm{n}=13)$, the MCT + Cel group $(\mathrm{n}=12)$ (Figure 1). To minimize potential confounders, the injection sequence of the two MCT groups was different every day and the order of measurements was also random. The grouping would not be announced until all data had been collected.

\section{Echocardiography}

The rats were anaesthetized with continuous isoflurane inhalation $(1.5-3 \%)$ and maintained with spontaneous respiration. Transthoracic echocardiography was performed by a $25 \mathrm{MHz}$ linear array transducer (Vevo 2100, VisualSonics, Toronto, Canada). Short axis M-mode recordings were obtained to measure left ventricle ejection fraction (LVEF). Pulsed-wave Doppler recordings of the right ventricular overflow tract were used to measure pulmonary acceleration time (PAT), peak ejection time (PET) and pulmonary artery velocity time integral (PAVTI). Tricuspid annular plane systolic excursion (TAPSE) was measured by using M-mode across the tricuspid valve annulus at the RV free wall. TAPSE was determined by quantifying the maximal longitudinal displacement of the lateral tricuspid valve annulus towards the RV apex during ventricular systole. Analyses were performed with observers blinded to the source of the images.

\section{$R V$ catheterization and hypertrophy index}

After echocardiography, terminal invasive haemodynamic measurements were performed to confirm RV pressure via $R V$ catheterization. The rats were anaesthetized with pentobarbital sodium $(40 \mathrm{mg} / \mathrm{kg})$ and fixed on plank. Then, the right jugular vein was isolated for intubation and lidocaine was use for local analgesia. The PE-50 tube was filled with heparin saline, connected to a pressure sensor (Techman, Chengdu, China) and inserted into the right external jugular vein. The appearance of the ventricular pressure wave indicated that the catheter reached the RV. Then the RV systolic pressure (RVSP) was analysed. After the RVSP was measured, the chest was quickly opened to harvest the heart and lungs of rats. The left and right atrium and blood vessels along the junction of the atrioventricular compartment were cut. Then the RV, left ventricle (LV) and interventricular septum (IVS) were separated and measured separately. The RV hypertrophy index was calculated as [RV/(LV + IVS)].

\section{Masson staining and haematoxylin-eosin (H\&E) staining}

After haemodynamic measurements and blood withdrawals, 
the heart and lungs were excised and harvested for fibrosis, morphometric and histologic analysis. The heart and middle lobe of the right lung were dissected and fixed in $4 \%$ paraformaldehyde for $24 \mathrm{~h}$, embedded in paraffin and sectioned. Heart sections were stained with Masson's trichrome and lung sections were stained with H\&E. A light microscope (Carl Zeiss, Jena, Germany) was used for overall histological assessment. RV fibrosis was assessed by Image-Pro Plus software (Version 6.0, Media Cybernetics, Silver Springs, MD, USA). Pulmonary arterial medial wall thickness (WT) was calculated by the following formula: WT $(\%)=\operatorname{area}_{\text {ext }}-\operatorname{area}_{\text {int }} /$ area $_{\text {ext }} \times 100$, where area $a_{\text {ext }}$ and area $_{\text {int }}$ are areas bounded by external and internal elastic lamina, respectively (19).

\section{Immunobistochemistry}

The expression of $\alpha$-smooth muscle actin ( $\alpha$-SMA) and CD68 in the middle lobe of the right lung was detected by immunohistochemistry staining. The tissue slides were deparaffinized with xylene and then serially rehydrated with ethanol. Following brief proteolytic digestion and peroxidase blocking, the slides were incubated with primary antibodies against $\alpha$-SMA (19245S, Cell Signaling Technology, USA, at a dilution of 1:500) and CD68 (GB11067, Servicebio, Wuhan, China, at a dilution of 1:500) at $4{ }^{\circ} \mathrm{C}$ overnight. The slides were first washed to remove the unbound primary antibodies and then incubated with a peroxidase conjugated secondary antibody. The specifically bound secondary antibody was detected with a DAKO EnVision detection System (Dako, Glostrup, Denmark).

\section{Western blot}

Flash-frozen inferior lobes of the right lungs were homogenized in radioimmunoprecipitation assay buffer supplemented with Halt protease and phosphatase inhibitor cocktail (Thermo Fisher Scientific, Waltham, MA, USA). Protein content was determined by using a BCA protein assay (Thermo Fisher Scientific, Waltham, MA, USA). Equivalent amounts of protein were resolved by SDS-polyacrylamide gel electrophoresis (PAGE) (10\%) and transferred to polyvinylidene difluoride membranes. After being blocked for $1 \mathrm{~h}$ in $5 \%$ nonfat dry milk and Tris-buffered saline, the membrane was incubated with the desired primary antibody for $8 \mathrm{~h}$ at $4^{\circ} \mathrm{C}$. The membrane was then treated with appropriate horseradish peroxidase conjugated secondary antibody (Cell Signaling
Technology, Danvers, MA, USA), and the immunoreactive bands were detected by chemiluminescence (ECL) reagents (Merck Millipore, Billerica, MA, USA). Specific antibodies against TGF- $\beta 1$ (sc-130348, Santa Cruz, CA, USA), IkB $\alpha$ (4814S, Cell Signaling Technology, Danvers, MA, USA), phosphorylated IKK $\alpha / \beta$ (2697S, Cell Signaling Technology, Danvers, MA, USA), p65 (sc-8008, Santa Cruz, CA, USA), and GAPDH (60004-1-lg, Proteintech, Chicago, IL, USA) were used in the immunoblot assay. For analysis, the expression of target proteins was normalized to GAPDH.

\section{Reverse transcription-quantitative polymerase chain reaction (RT-qPCR)}

The superior lobes of the right lungs were isolated from the rats and homogenized, and total RNA was extracted with TRIzol reagent (Thermo Fisher Scientific, Waltham, MA, USA) according to the manufacturer's instructions. One thousand nanograms of RNA was reverse-transcribed to cDNA by using the PrimeScript ${ }^{\mathrm{TM}} \mathrm{RT}$ reagent Kit (RR037A, Takara, Tokyo, Japan). The PCR primers were designed and synthesized by Invitrogen (Thermo Fisher Scientific, Waltham, MA, USA). The following specific primers were generated and used: for Collagen I, forward primer: GTACATCAGCCCAAACCCCA, reverse primer: TCGCTTCCATACTCGAACTGG; for Collagen III, forward primer: TGGTGGCTTTC AGTTCAGCTA, reverse primer: ATTGCCATTGGCC TGATCCA; for TGF- $\beta 1$, forward primer: CTGCTGAC CCCCACTGATAC, reverse primer: AGCCCTGTATT CCGTCTCCT; for monocyte chemotactic protein 1 (MCP-1), forward primer: TGTCTCAGCCA GATGCAGTT, reverse primer: CAGCCGACTCAT TGGGATCA; for IL-1 $\beta$, forward primer: TAGCAGC TTTCGACAGTGAGG, reverse primer: CTCCACGGG CAAGACATAGG; for IL-6, forward primer: CATTCT GTCTCGAGCCCACC, reverse primer: GCTGGAAGT CTCTTGCGGAG; for IL-10, forward primer: CCTGGT AGAAGTGATGCCCC, reverse primer; GACACCTT TGTCTTGGAGCTTAT; for GAPDH, forward primer: CGCTAACATCAAATGGGGTG, reverse primer: CG CTAACATCAAATGGGGTG. Quantitative PCR was performed using TB Green ${ }^{\circledR}$ Premix Ex Taq ${ }^{\text {TM }}$ II (RR820A, Takara, Tokyo, Japan), and the samples were assayed in duplicate according to the manufacturer's instructions in a BioRad CFX96 PCR system (Bio-Rad, Hercules, CA, USA). For analysis, the expression of target genes was normalized 
to GAPDH.

\section{Cell culture}

Human pulmonary artery smooth cells (HPASMCs, ScienCell, Carlsbad, CA, USA) were cultured at $37^{\circ} \mathrm{C}$ and $5 \% \mathrm{CO}_{2}$ with Dulbecco's modified Eagle's medium (DMEM) containing 10\% fetal bovine serum (FBS) and $1 \%$ penicillin/solution (Gibco, Grand Island, NY, USA). For in vitro experiments, HPASMCs between passages 3-8 were used.

\section{EdU proliferation assay}

Cell proliferation was studied with an EdU Cell Proliferation Assay Kit (EdU-488, Beyotime, Shanghai, China), according to the manufacturer's protocol. HPASMCs were seeded into 24-well plates at a concentration of $1.8 \times 10^{4}$ cells/well and cultured overnight. Then, the medium was replaced, $0.5 \mu \mathrm{M}$ or $1.0 \mu \mathrm{M}$ celastrol was added to the medium, and HPASMCs were exposed to hypoxic ( $2 \%$ oxygen) or normoxic $(21 \%$ oxygen) conditions at $37^{\circ} \mathrm{C}$ for $36 \mathrm{~h}$. Then, $10 \mu \mathrm{M} \mathrm{EdU}$ was added to the cells. At the end of the 4-hour incubation, the medium was removed, and the cells were fixed in $4 \%$ paraformaldehyde for $15 \mathrm{~min}$ at room temperature. After aspiration of the fixing solution, the cells were stained with the BeyoClick ${ }^{\mathrm{TM}}$ EdU Cell Proliferation Kit with Alexa Fluor 488 and DAPI (nuclear stain). Then the results were visualized through a fluorescence microscope (Leica, Wetzlar, Germany). The results were quantified by using Image-Pro Plus software (Version 6.0, Media Cybernetics, Silver Springs, MD, USA).

\section{Cell viability assay}

Cell viability was assessed by using Cell Counting Kit- 8 (CCK-8, Beyotime, Shanghai, China). In brief, HPASMCs were seeded into 96-well plates at a concentration of $6 \times 10^{3}$ cells/well and cultured for $24 \mathrm{~h}$. Then, the medium was replaced, 0.5 or $1.0 \mu \mathrm{M}$ celastrol was added to the medium, and HPASMCs were exposed to hypoxic ( $2 \%$ oxygen) or normoxic $\left(21 \%\right.$ oxygen) conditions at $37{ }^{\circ} \mathrm{C}$ for $36 \mathrm{~h}$. After exposure and replacement of the medium, $10 \mu \mathrm{L}$ CCK8 solution was added to each well and incubated at $37{ }^{\circ} \mathrm{C}$ for another $3 \mathrm{~h}$. The optical density value at a wavelength of $450 \mathrm{~nm}$ was determined by using a microplate reader (Thermo Fisher Scientific, Waltham, MA, USA).

\section{Apoptosis assay}

Cell apoptosis was detected using a FITC Annexin V Apoptosis Detection Kit (BD Biosciences, San Jose, CA, USA) according to the manufacturer's instructions. After expose to hypoxia ( $2 \%$ oxygen) or normoxia ( $21 \%$ oxygen) at $37^{\circ} \mathrm{C}$ for $36 \mathrm{~h}$, the HPASMCs seeded in 6-well plates were harvested and washed twice with $4{ }^{\circ} \mathrm{C}$ phosphate buffer solution. Then, the cells were resuspended in binding buffer at a concentration of $1 \times 10^{6}$ cells $/ \mathrm{mL}$ and incubated with $5 \mu \mathrm{L}$ FITC Annexin $\mathrm{V}$ at $25^{\circ} \mathrm{C}$ for $15 \mathrm{~min}$ in the dark. After incubation, $5 \mu \mathrm{L}$ PI was added. Then the samples were analysed using a flow cytometer (BD Biosciences, San Jose, CA, USA) within $1 \mathrm{~h}$.

\section{Statistical analyses}

All the data were presented as the mean \pm SD. All the statistical analyses were performed by using GraphPad Prism software (Version 8, La Jolla, CA, USA). Comparisons among groups were assessed with one-way ANOVA followed by Tukey's post hoc test. Differences were considered statistically significant if $\mathrm{P}<0.05$.

\section{Ethical statement}

Experiments were performed under a project license (Nos. 2020000093) granted by the Institutional Animal Use and Care Committee of Sun Yat-sen University, Guangzhou City, Guangdong Province, China, in compliance with the national or institutional guidelines for the care and use of animals. A protocol was prepared before the study without registration.

\section{Results}

\section{Celastrol improves $R V$ function and reduces RVSP in $\mathrm{PAH}$ rats}

Four weeks after MCT injection, PAH rat models were established. There was no statistical difference in PET between different groups (Normal: $83.96 \pm 6.20 \mathrm{~ms} v \mathrm{~s}$. MCT + Veh: $90.24 \pm 9.65 \mathrm{~ms} v s . \mathrm{MCT}+\mathrm{Cel}: 93.01 \pm 5.35 \mathrm{~ms}, \mathrm{P}>0.05$ ) (Figure 2A), however, significantly reduced PAT (Normal: $38.68 \pm 6.41 \mathrm{~ms} v s$. MCT + Veh: $23.40 \pm 3.74 \mathrm{~ms} v s$. MCT + Cel: $32.00 \pm 5.83 \mathrm{~ms}, \mathrm{P}<0.05)$, PAT/PET (Normal: $0.46 \pm 0.06$ vs. MCT + Veh: $0.26 \pm 0.04$ vs. MCT + Cel: $0.34 \pm 0.05$, $\mathrm{P}<0.05$ ) and TAPSE (Normal: $1.91 \pm 0.26 \mathrm{~mm} v s . \mathrm{MCT}+$ Veh: $1.20 \pm 0.21 \mathrm{~mm}$ vs. MCT + Cel: $1.49 \pm 0.24 \mathrm{~mm}, \mathrm{P}<0.05)$ 
A

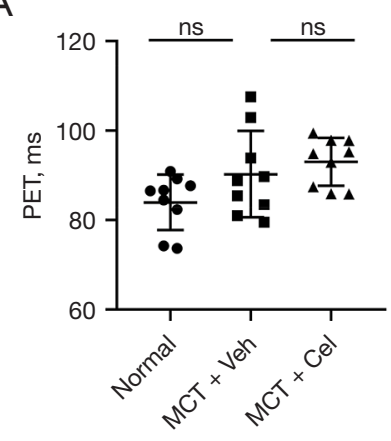

D

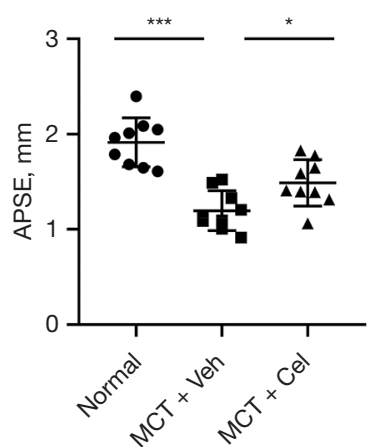

G

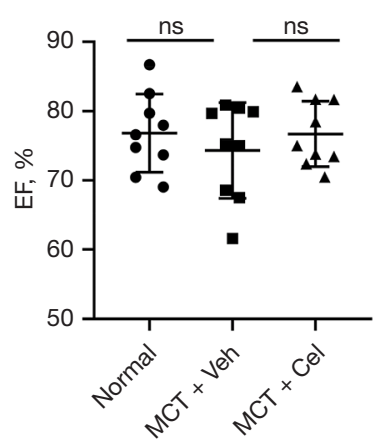

B

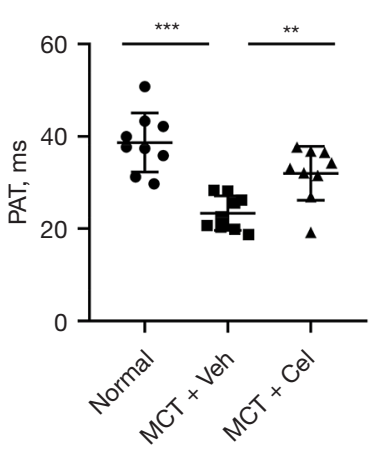

$\mathrm{E}$

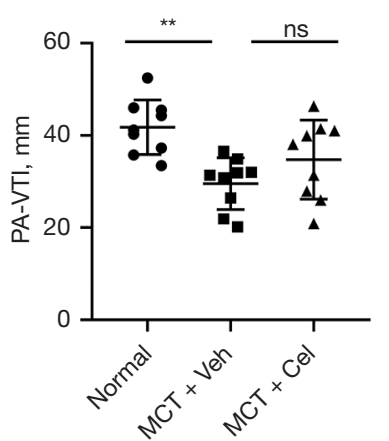

$\mathrm{H}$

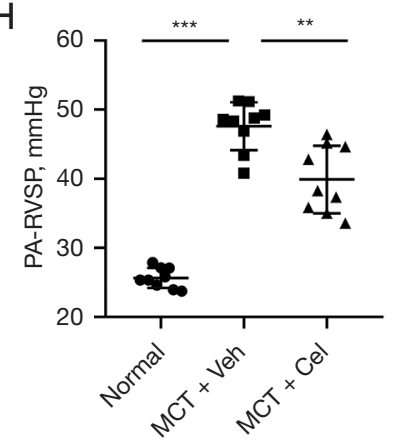

C

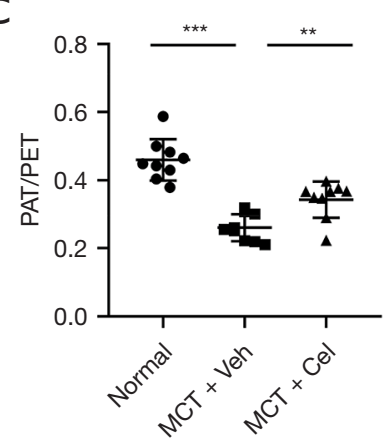

$\mathrm{F}$

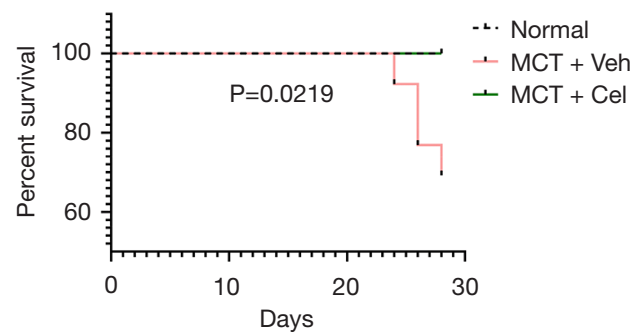

Figure 2 Celastrol treatment improves RV function and reduces RVSP in PAH rats. Quantification of PET (A), PAT (B), PAT/PET (C), TAPSE (D), PA-VTI (E), LVEF (G), RVSP (H) four weeks after MCT injection (n=9 for each group); (F) Kaplan-Meier survival curves in the different groups ( $\mathrm{n}=10$ for the normal group, $\mathrm{n}=13$ for the vehicle group, $\mathrm{n}=12$ for the celastrol group). ${ }^{*}, \mathrm{P}<0.05,{ }^{* *}, \mathrm{P}<0.01,{ }^{* * *}, \mathrm{P}<0.001$. RV, right ventricle; RVSP, RV systolic pressure; PAH, pulmonary arterial hypertension; PET, peak ejection time; TAPSE, tricuspid annular plane systolic excursion; MCT, monocrotaline.

were observed in the PAH rats compared with the normal rats, which confirmed RV dysfunction (Figure $2 B-2 D$ ). Treatment with celastrol significantly improved the RV function of PAH rats relative to treatment with vehicle (Figure 2B-2D). The PAT-VTI of the vehicle group decreased significantly compared to that of the normal group (Normal: $41.79 \pm 5.90 \mathrm{~mm} v s$. MCT + Veh: $29.58 \pm 5.60 \mathrm{~mm}$, $\mathrm{P}<0.01$ ) (Figure $2 E$ ). Although the celastrol group had increased PAT-VTI compared to the vehicle group (MCT + Cel: $34.77 \pm 8.53 \mathrm{~mm} v s$. MCT + Veh: $29.58 \pm 5.60 \mathrm{~mm}, \mathrm{P}>0.05$ ), there was no significant difference (Figure 2E). Furthermore, four rats died before the end of the study. All of them were from the vehicle group. Necropsy of the dead animals did not reveal a clear cause of death. However, celastrol treatment completely prevented mortality (Figure $2 F$ ). As we expected, LVEF had no significant change in any of the groups (Normal: $76.84 \% \pm 5.65 \%$ vs. MCT + Veh: $74.32 \% \pm 6.92 \%$ vs. MCT + Cel: $76.73 \% \pm 4.73 \%, \mathrm{P}>0.05$ ) (Figure $2 G$ ), meaning neither MCT nor celastrol had any effect on LV systolic function. To explore the role of celastrol in the development of PAH, we 
A

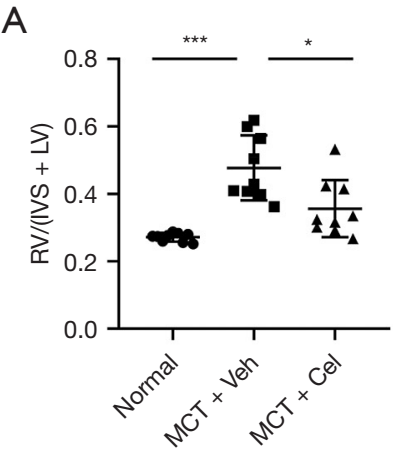

C

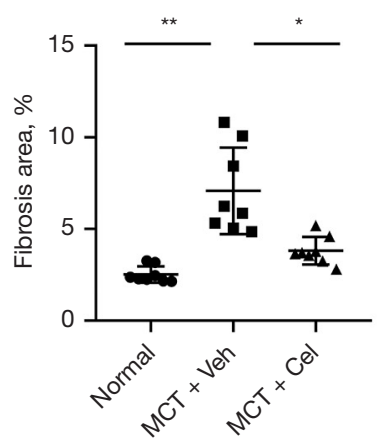

B
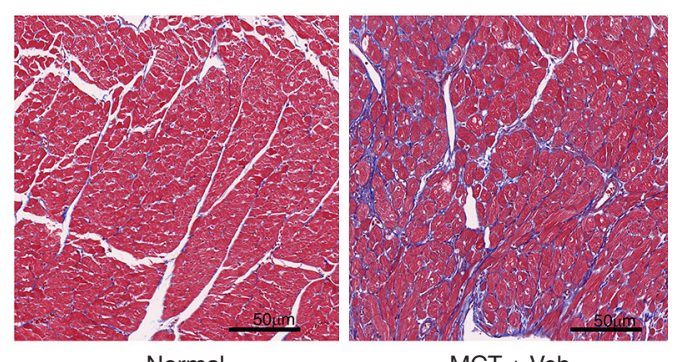

$\mathrm{MCT}+$ Veh

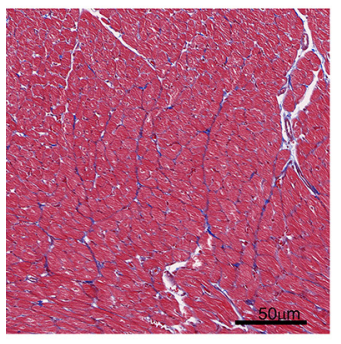

$\mathrm{MCT}+\mathrm{Cel}$
D

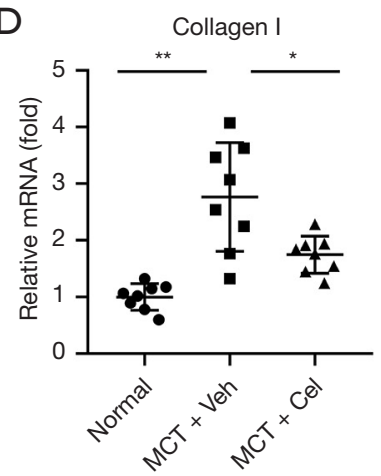

$\mathrm{E}$

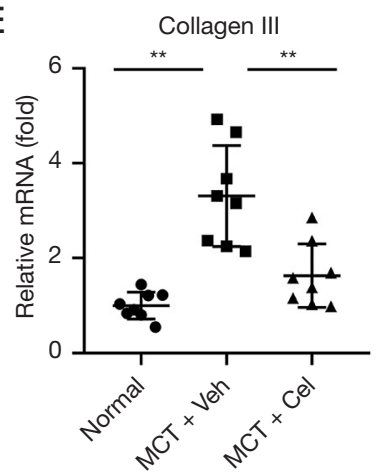

Figure 3 Celastrol alleviates RV hypertrophy and fibrosis in PAH rats. (A) RV hypertrophy index; (B) Histopathological features of collagen deposition based on Masson staining of heart sections ( $\times 40)$; (C) Quantification of Masson staining; (D), (E) RV mRNA expression of collagen I and collagen III. $\mathrm{n}=8$ for each group. ${ }^{*}, \mathrm{P}<0.05,{ }^{* *}, \mathrm{P}<0.01,{ }^{* *}, \mathrm{P}<0.001$. RV, right ventricle; PAH, pulmonary arterial hypertension. MCT, monocrotaline.

examined RVSP with a pressure catheter that can represent pulmonary artery pressure more accurately. As shown in Figure $2 \mathrm{H}$, celastrol significantly attenuated $\mathrm{PAH}$ by reducing RVSP to $83.82 \%($ MCT + Cel: $39.91 \pm 4.88 \mathrm{mmHg} v$ s. MCT + Veh: $47.62 \pm 3.46 \mathrm{mmHg}, \mathrm{P}<0.01)$.

\section{Celastrol alleviates $R V$ bypertrophy and fibrosis in $P A H$ rats}

Due to the increased RV pressure in PAH rats, the RV was remodeled and enlarged, which was shown as a higher $\mathrm{RV} /(\mathrm{LV}+\mathrm{IVS}$ ) ratio (Normal: $0.27 \pm 0.01 v s . \mathrm{MCT}+\mathrm{Veh}$ : $0.48 \pm 0.10$ vs. MCT + Cel: $0.36 \pm 0.08, \mathrm{P}<0.05)$. However, the celastrol group had a significantly lower $\mathrm{RV} /(\mathrm{LV}+\mathrm{IVS})$ ratio than the vehicle group (MCT + Cel: $0.36 \pm 0.08 v s$. MCT + Veh: $0.48 \pm 0.10, \mathrm{P}<0.05$ ) (Figure $3 A$ ). Using Masson staining, we found a 2.82 -fold increase in the volume fraction of fibrosis in the RV in vehicle rats compared with normal rats (Normal: $2.51 \% \pm 0.45 \%$ vs. MCT + Veh: $7.08 \% \pm 2.37 \%$, $\mathrm{P}<0.01)$. Treatment with celastrol significantly reduced RV fibrosis (MCT + Cel: $3.82 \% \pm 0.75 \%$ vs. MCT + Veh: $7.08 \% \pm 2.37 \%, \mathrm{P}<0.05$ ) (Figure 3B,3C). Similarly, the
mRNA expression of fibrosis-related molecules in the RV, collagen I (Normal: $1.00 \pm 0.23$ vs. MCT + Veh: $2.76 \pm 0.96$, $\mathrm{P}<0.01$ ) and collagen III (Normal: $1.00 \pm 0.28$ vs. MCT + Veh: $3.31 \pm 1.06, \mathrm{P}<0.01)$ increased in PAH rats. Celastrol treatment significantly reduced the expression of these molecules (collagen I, MCT + Cel: $1.75 \pm 0.33$ vs. MCT + Veh: $2.76 \pm 0.96, \mathrm{P}<0.05$; collagen III, MCT + Cel: $1.63 \pm 0.67$ vs. MCT + Veh: $3.31 \pm 1.06, \mathrm{P}<0.01$ ) (Figure $3 D, 3 E$ ).

\section{Celastrol attenuates pulmonary vascular remodeling}

MCT injection induced remodeling of the pulmonary vasculature, as evidenced by increased wall thickness of the small pulmonary arteries (PA wall thickness) with an outer diameter between 50 and $100 \mu \mathrm{m}$ (Normal: $1.00 \pm 0.14 \mathrm{vs}$. MCT + Veh: $2.10 \pm 0.23, \mathrm{P}<0.001)$. Celastrol treatment led to a modest suppression of MCT-induced PA wall thickness (MCT + Cel: $1.76 \pm 0.19$ vs. MCT + Veh: $2.10 \pm 0.23, \mathrm{P}<0.05$ ) (Figure $4 A, 4 B$ ). Immunohistochemistry of $\alpha$-SMA showed increased pulmonary artery muscularization in small pulmonary arteries with an outer diameter between 50 and 
A

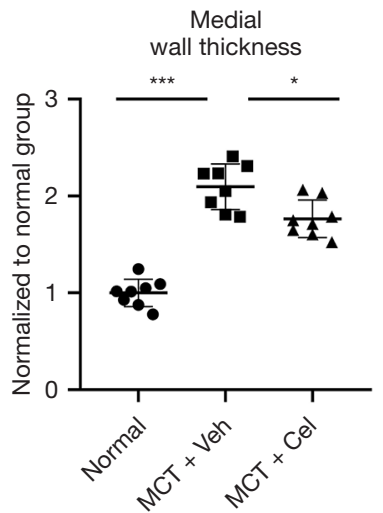

C

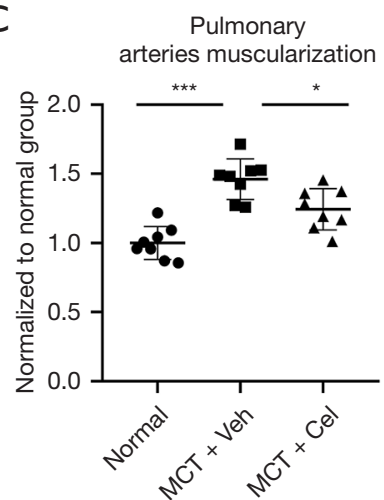

$\mathrm{E}$

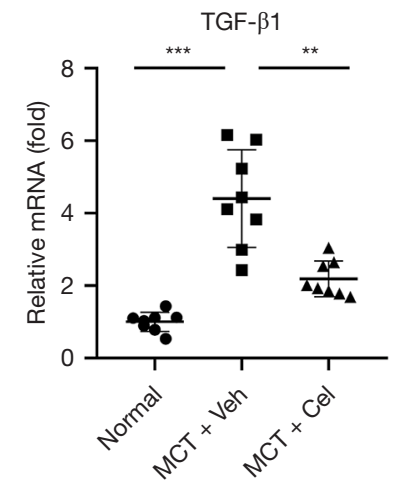

B

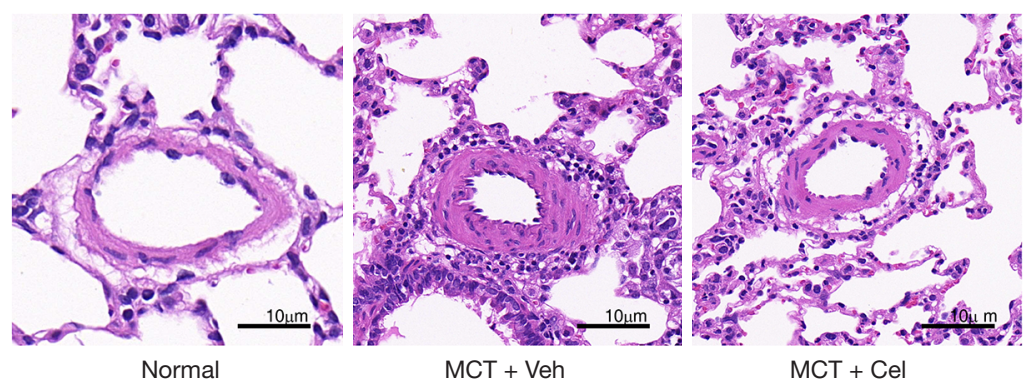

D

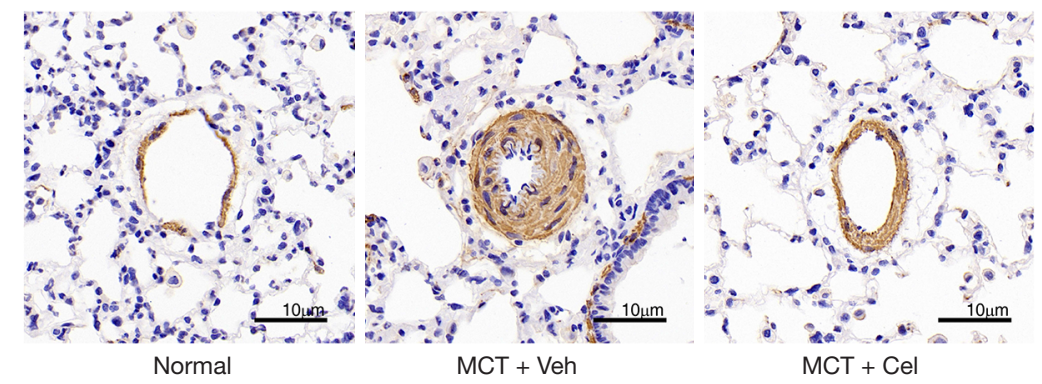

$\mathrm{F}$

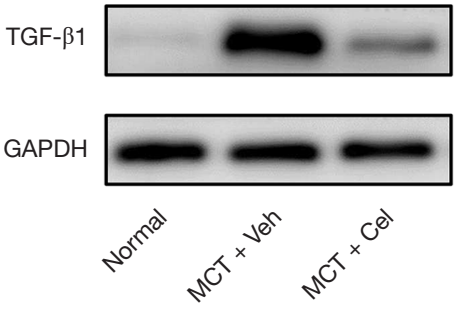

G

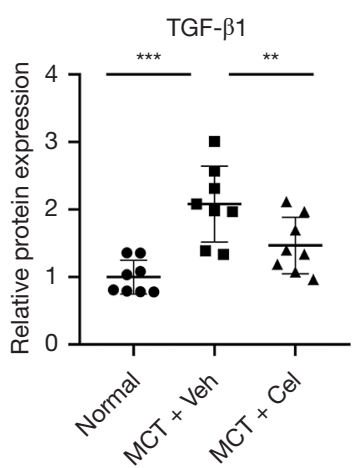

Figure 4 Celastrol attenuates pulmonary vascular remodeling. (A) Quantification of the medial wall thickness of the small pulmonary arteries with an outer diameter between 50 and $100 \mu \mathrm{m}$. (B) Photomicrographs of lung tissue sections stained with H\&E (x99); (C) Quantification of the arteries muscularization of the small pulmonary arteries with an outer diameter between 50 and $100 \mu \mathrm{m}$; (D) Expression of $\alpha$-SMA in lungs evaluated with immunohistochemistry staining ( $\times 99$ ); (E) mRNA expression of TGF- $\beta 1$ in lung tissues; (F) Western blots of TGF- $\beta 1$ in lung tissues; (G) Quantification of the TGF- $\beta 1$ expression in lung tissues. $\mathrm{n}=8$ for each group. * $\mathrm{P}<0.05$, ${ }^{* *}, \mathrm{P}<0.01$, ${ }^{* * *}, \mathrm{P}<0.001$. MCT, monocrotaline.

$100 \mu \mathrm{m}$ in vehicle rats, whereas this effect was weakened by celastrol treatment (Normal: $1.00 \pm 0.12$ vs. MCT + Veh: $1.46 \pm 0.15$ vs. MCT + Veh: $1.24 \pm 0.15, \mathrm{P}<0.05)$ (Figure $4 C, 4 D$ ).
Furthermore, celastrol significantly reduced the expression of TGF- $\beta 1$ in the superior lobe of the right lung at the

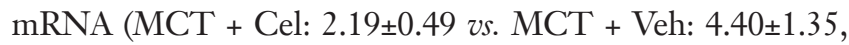


$\mathrm{P}<0.01)$ and protein levels $(\mathrm{MCT}+\mathrm{Cel}: 1.51 \pm 0.43$ vs. $\mathrm{MCT}+\mathrm{Veh}: 2.14 \pm 0.58, \mathrm{P}<0.05)$ compared with vehicle rats (Figure 4E-4G).

\section{Celastrol inbibits inflammation in lungs}

Previous studies have demonstrated that elevated macrophage infiltration and enhanced levels of inflammatory cytokines are correlated with the severity of PAH (7). We therefore wondered whether celastrol treatment affected the infiltration of macrophages and the production of inflammatory cytokines in vivo. As shown in Figure $5 A, 5 B$, celastrol treatment markedly decreased the number of macrophages in the lungs of PAH rats compared with vehicle treated PAH rats $(\mathrm{MCT}+$ Cel: $202.88 \pm 51.43$ vs. MCT + Veh: 445.625 $\pm 132.93, \mathrm{P}<0.01)$. RT-qPCR revealed that the expression levels of several proinflammatory cytokines, including MCP- $1, \mathrm{IL}-1 \beta$ and IL6 , in the lungs were significantly increased in the vehicle group compared with the normal group, but they were significantly inhibited by celastrol treatment (MCP-1, Normal: $1.00 \pm 0.39$ vs. MCT + Veh: $3.46 \pm 0.97$ vs. MCT + Cel: $2.21 \pm 0.81$; IL-1 $\beta$, Normal: $1.00 \pm 0.33$ vs. MCT + Veh: $6.07 \pm 1.75$ vs. MCT + Cel: $3.45 \pm 1.21$; IL-6, Normal: $1.00 \pm 0.52$ vs. MCT + Veh: $5.51 \pm 1.96$ vs. MCT + Cel: $2.67 \pm 1.13$, all $\mathrm{P}<0.05$ ) (Figure 5C-5E). Celastrol further remarkably increased the expression of IL-10 (MCT + Cel: $0.68 \pm 0.14$ vs. MCT + Veh: $0.50 \pm 0.11, \mathrm{P}<0.05)$, which is an anti-inflammatory factor, in the lungs of rats with PAH (Figure $5 F)$.

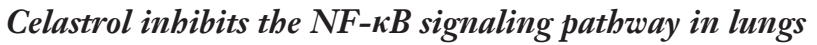

Given that the NF- $\kappa \mathrm{B}$ signaling pathway plays a crucial role in PAH initiation and progression $(8,9)$, we next tested whether celastrol inhibited inflammation through the $\mathrm{NF}-\mathrm{kB}$ signaling pathway. In PAH rats, the lung NF- $\mathrm{\kappa B}$ signaling pathway was active (Figure $5 G-57$ ), as shown by increased degradation of I B $\alpha$ (Normal: $1.00 \pm 0.19 v s$. MCT + Veh: $0.60 \pm 0.15, \mathrm{P}<0.01)$, phosphorylation of IKK $\alpha / \beta$ (Normal: $1.00 \pm 0.16$ vs. MCT + Veh: $1.94 \pm 0.37, \mathrm{P}<0.001$ ) and expression of p65 (Normal: $1.00 \pm 0.38$ vs. MCT + Veh: $1.86 \pm 0.63, \mathrm{P}<0.01)$. However, celastrol treatment significantly antagonized the effects of MCT in rat lungs (I B $\alpha$, MCT + Cel: $0.83 \pm 0.14$ vs. MCT + Veh: $0.60 \pm 0.15$, $\mathrm{P}<0.05 ; \mathrm{p}-\mathrm{IKK} \alpha / \beta, \mathrm{MCT}+\mathrm{Cel}: 1.46 \pm 0.24$ vs. MCT + Veh: $1.94 \pm 0.37, \mathrm{P}<0.01 ; \mathrm{p} 65, \mathrm{MCT}+\mathrm{Cel}: 1.30 \pm 0.25$ vs. MCT + Veh: $1.86 \pm 0.63, \mathrm{P}<0.05)$.

\section{Celastrol inhibits the proliferation of HPASMCs in response to bypoxia}

The proliferation of PASMCs contributes to the progression of vascular remodeling which leads to irreversible progression of PAH (20). We thus examined the effect of celastrol treatment on the proliferative potential of cultured HPASMCs. EdU proliferation assay showed that hypoxia promoted HPASMCs proliferation, but celastrol exhibited antiproliferation effects on HPASMCs at both low and high concentrations ( 0.5 and $1.0 \mu \mathrm{M}$ ) under hypoxia (Normoxia: $1.00 \pm 0.25$ vs. Hypoxia: $2.08 \pm 0.19 v s$. Нypoxia $+0.5 \mu \mathrm{M}: 1.59 \pm 0.42 v s$. Нypoxia $+1.0 \mu \mathrm{M}$ : $1.19 \pm 0.28, \mathrm{P}<0.05$ ) (Figure $6 A, 6 B$ ). However, the CCK-8 assay showed that low concentrations of celastrol had tendency to enhance the cell activity of HPASMCs under hypoxia without a significant difference, but high concentrations of celastrol had a notable inhibitory effect (Normoxia: $1.00 \pm 0.04$ vs. Hypoxia: $1.37 \pm 0.11$ vs. Hypoxia $+0.5 \mu \mathrm{M}$ : $1.46 \pm 0.15$ vs. Hypoxia $+1.0 \mu \mathrm{M}: 1.19 \pm 0.11, \mathrm{P}<0.05$ ) (Figure 6C). An apoptosis assay was further performed to observe the effect of celastrol on HPASMCs apoptosis. As shown in Figure 6D,6E, no significant proapoptotic effect was observed, which demonstrated that both 0.5 and $1.0 \mu \mathrm{M}$ celastrol had no obvious toxicity to HPASMCs (Normoxia: $8.37 \% \pm 1.42 \% v$ s. Hypoxia: $7.19 \% \pm 1.48 \%$ vs. Hypoxia $+0.5 \mu \mathrm{M}: 7.73 \% \pm 1.63 \%$ vs. Hypoxia $+1.0 \mu \mathrm{M}: 8.64 \% \pm 2.05 \%, \mathrm{P}>0.05)$.

\section{Discussion}

Using a universally adopted model of PAH, we found that treatment with celastrol for 4 weeks significantly improved RV function and decreased pulmonary artery pressure. We also demonstrated that these improvements were associated with decreased $\mathrm{RV}$ and pulmonary vascular remodeling, and reduced lung macrophage infiltration and inflammation. Furthermore, we found that celastrol inhibits the proliferation of HPASMCs under hypoxic conditions in vitro. Finally, we demonstrated that the decreased remodeling of the pulmonary vasculature may be related to the inhibition of the NF- $\kappa \mathrm{B}$ signaling pathway.

Among preclinical models of PAH, the MCT animal model offers the advantage of mimicking several key aspects of human PAH, including vascular remodeling, smooth muscle cell proliferation, inflammatory cytokine upregulation, and RV remodeling and failure (21), although it still cannot perfectly mimic human PAH. Four weeks 


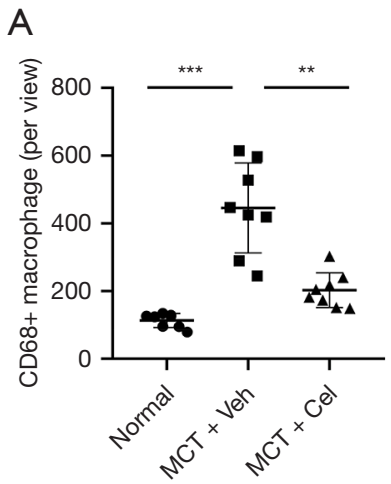

C

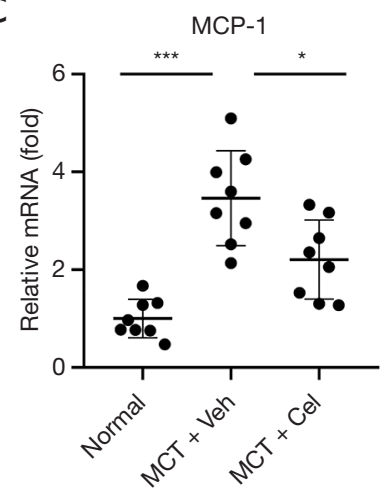

G

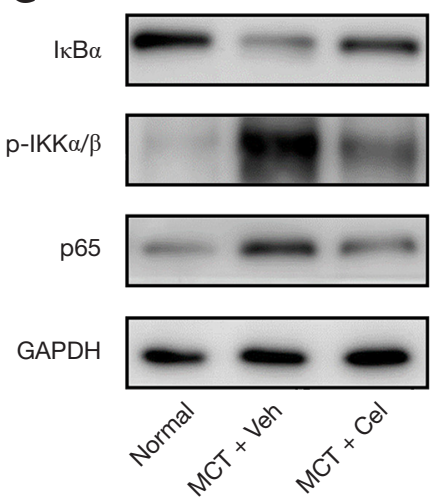

B

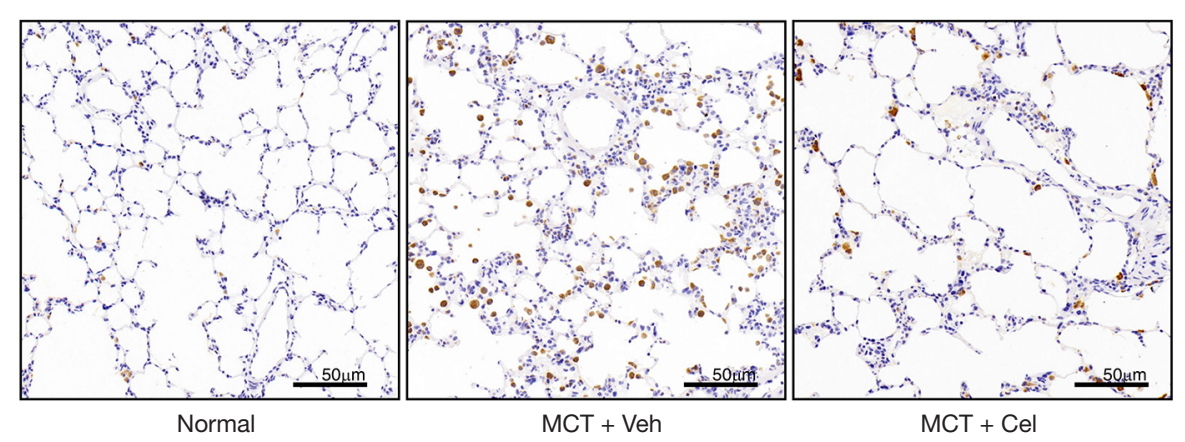

D
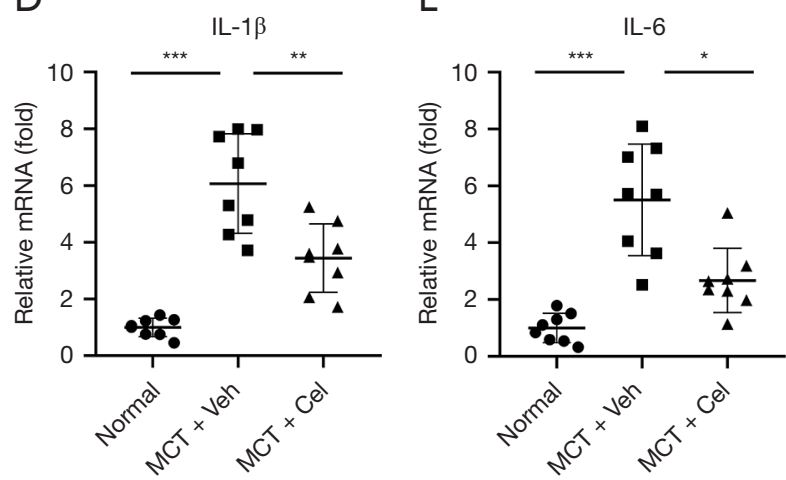

$\mathrm{H}$

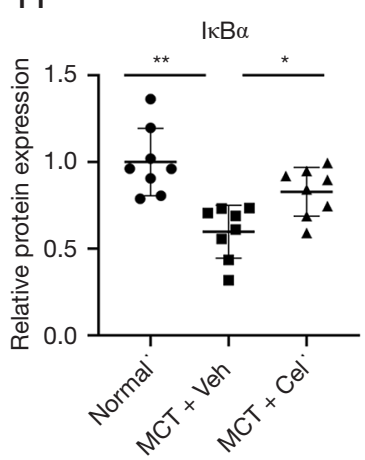

E

F

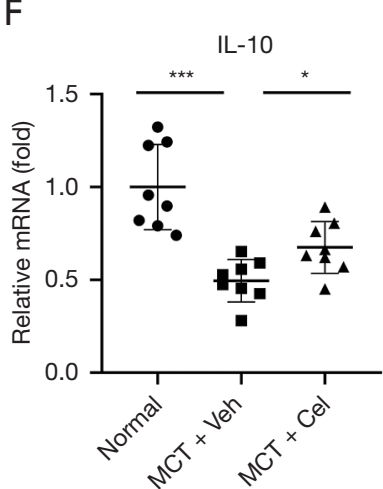

I
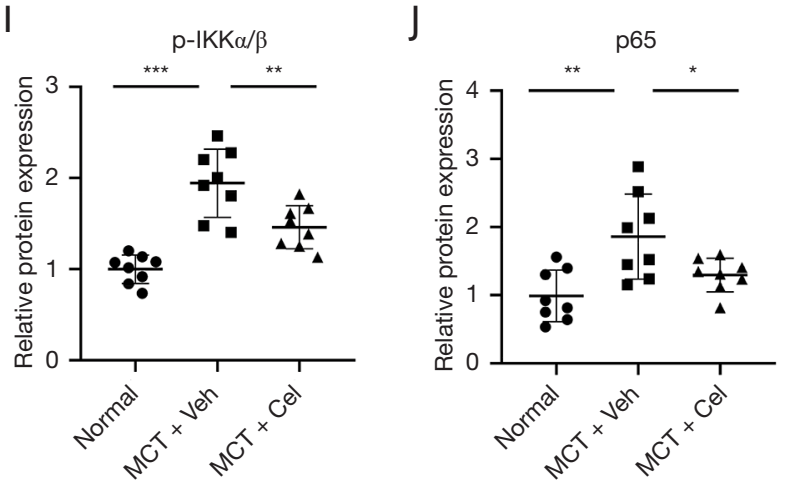

Figure 5 Celastrol inhibits the inflammatory and NF-kB signaling pathway in the lungs of PAH rats. (A) Quantification of CD68 positive cells per view; (B) Representative immunohistochemistry staining images of CD68 positive cells in lung tissues ( $\times 20)$; (C-F) mRNA expression of MCP-1,

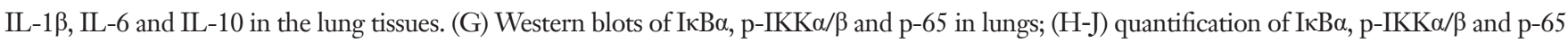
expression in the lungs. $\mathrm{n}=8$ for each group. ${ }^{*}, \mathrm{P}<0.05,{ }^{* *}, \mathrm{P}<0.01,{ }^{* * *}, \mathrm{P}<0.001$. $\mathrm{PAH}$, pulmonary arterial hypertension; MCT, monocrotaline.

after MCT injection, increased pulmonary artery pressure resulted in dysfunction of the RV. Prolonged PAT time and increased PAT/PET ratio and TASPE all demonstrated a protective effect of celastrol on RV function, which may explain the lack of premature death of the celastrol treated rats. The results of Masson staining and mRNA expression of collagen I and collagen III in RV suggested serious RV remodeling in PAH induced by MCT, which was alleviated by celastrol treatment. Unfortunately, we did not apply celastrol to a pulmonary trunk banding (PTB) rat model. Therefore, even though we found that celastrol can reduce RV remodeling caused by $\mathrm{PAH}$, we 
A

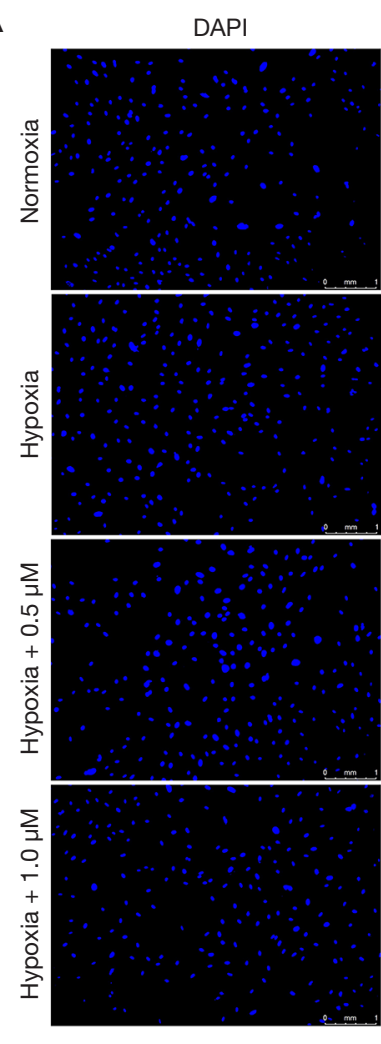

D

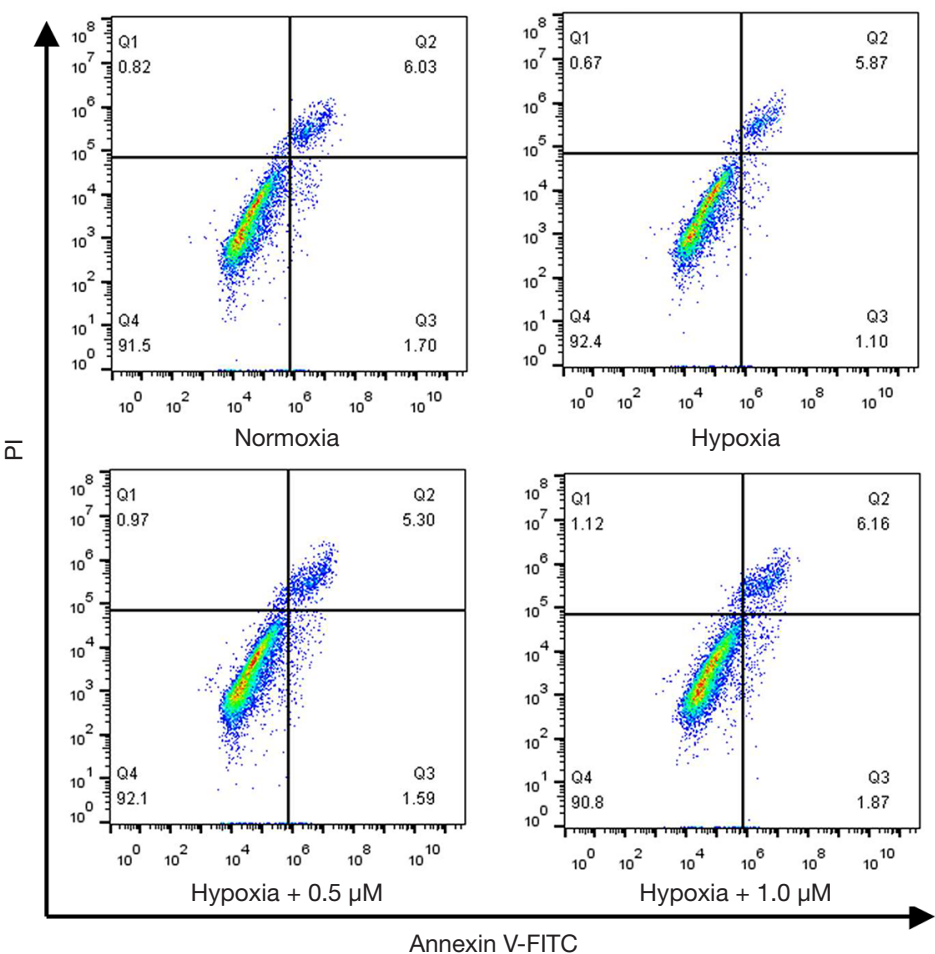

B

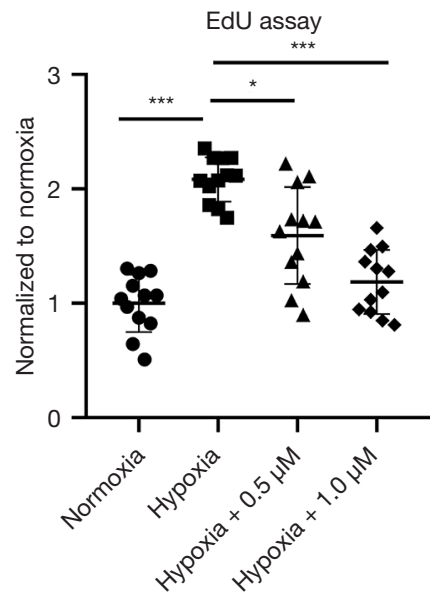

C

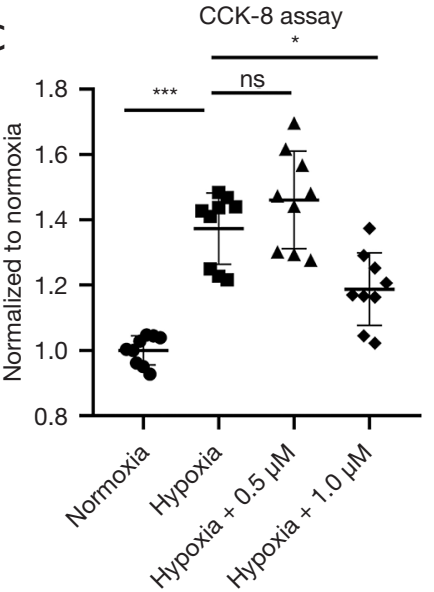

$E$

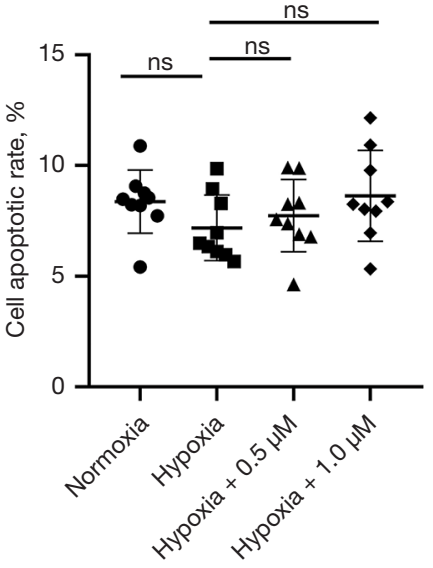

Figure 6 Celastrol inhibits the proliferation of HPASMCs in response to hypoxia in vitro. (A) Representative images of the EdU assay of HPASMCs in vitro ( $\times 100$ ); (B) and (C) quantification of the EdU assay and CCK-8 assay of HPASMCs; (D) HPASMCs apoptosis in FITC Annexin V flow cytometry; (E) the apoptosis ratio as calculated with the early and late-stage apoptotic cells ( $\mathrm{n}=3$, biological replicates; 4 wells each time for the EdU assay, 3 wells each time for the CCK-8 assay, 3 wells each time for the apoptosis assay). ${ }^{*}, \mathrm{P}<0.05,{ }^{* *}, \mathrm{P}<0.01,{ }^{* * *}, \mathrm{P}<0.001$. HPASMCs, human pulmonary artery smooth cells. 
still didn't know the precise mechanism. Whether celastrol directly reduces lung remodeling and pulmonary vascular resistance and then indirectly relieves $\mathrm{RV}$ remodeling, or whether celastrol directly inhibits RV fibroblasts to ease RV remodeling, or celastrol works in both directions remains to be verified. However, Ye et al. (18) studies clarified that celastrol could directly bind to and inhibit signal transducer and activator of transcription-3 (STAT3) phosphorylation and nuclear translocation to alleviate left ventricular hypertrophy and fibrosis in a pressure overload model in mice. Correspondingly, celastrol was recently found to directly ameliorate RV fibrosis in a PTB mouse model (22). We, therefore, speculated that celastrol protected RV function not only by reducing the afterload of the RV but also by directly inhibiting RV remodeling. Overall, future experimental studies should further clarify the molecular mechanisms of celastrol on RV in the PTB model.

Over the past two decades, inflammatory processes have been recognized as major pathogenic components of PAH (23). Recently, increasing evidence has indicated that macrophages play an important role in pulmonary vascular remodeling in PAH. Macrophage infiltration is not only a characteristic hallmark, but also a critical component of pulmonary vascular remodeling associated with PAH (24). Abid et al. (25) found that macrophages and PASMCs communicated to promote PASMCs proliferation via CCR2 (receptor of MCP-1) and CCR5, and targeting CCR2, CCR5 or both can prevent or reverse PAH in mice. In a rat arthritis model, celastrol was found to significantly decrease the number of $\mathrm{CD} 68^{+}$macrophages and the overall inflammatory cellularity in arthritic synovial tissue without side effects (26). Consistent with these findings, our results suggested that celastrol therapy significantly reduced pulmonary macrophage infiltration and decreased the MCP-1 mRNA expression induced by MCT, which may partly explain the reduced pulmonary vascular remodeling in celastrol rats. In addition to macrophages infiltration, the upregulated expression of proinflammatory cytokines and downregulated expression of anti-inflammatory cytokines are also characteristics of $\mathrm{PAH}$ and further promote its development (27). For instance, elevated IL-6 levels played critical roles in mouse hypoxia-induced $\mathrm{PAH}$ by promoting the polarization of macrophages in lungs (28). In this study, we found that celastrol treatment significantly reduced the expression of the proinflammatory cytokines IL-1 $\beta$, IL-6, and TGF- $\beta 1$ in the lungs of rats with PAH and increased the expression of the anti-inflammatory cytokine IL-10.

The NF- $\kappa \mathrm{B}$ signaling pathway regulates the expression of a wide variety of genes involved in cell proliferation, inflammation, survival, migration, and angiogenesis $(29,30)$. Thus, the NF-KB signaling pathway has long been considered holy grail as a target for new anti-inflammatory drugs (31). Sethi et al. (32) found that celastrol could apparently inhibit the TNF- $\alpha$-induced I $\kappa$ Ba kinase activation, IкBa phosphorylation, IкBa degradation, p65 nuclear translocation and phosphorylation, and NF- $\mathrm{KB}-$ mediated reporter gene expression. Importantly, celastrol was also found to inhibit IKK activity and constitutively active IKK $\beta$ activity in a dose dependent manner without directly suppressing the DNA binding ability of NF- $\mathrm{B}$ (33). Consistent with previous studies, our study confirmed that celastrol can indeed inhibit the activation of the NF- $\kappa \mathrm{B}$ signaling pathway in the lungs of $\mathrm{PAH}$ rats. Considering the critical role of inflammation in the MCT-induced $\mathrm{PAH}$ and the strong connection between inflammation and $\mathrm{NF}-\kappa \mathrm{B}$ signaling pathway $(23,31)$, the inhibition of the NF- $\kappa B$ signaling pathway might explain the reduction in lung inflammation and less severe pulmonary vascular remodeling in celastrol treated rats. Several published reports within the last decade have shown that activation of the NF- $\kappa \mathrm{B}$ signaling pathway promotes cell survival and proliferation and that downregulation of the NF- $\mathrm{NB}$ signaling pathway sensitizes cells to apoptosis induction $(34,35)$. Furthermore, celastrol reportedly has anti-cancer effects both in vitro and in vivo (36). For example, Guo et al. (37) recently found that celastrol induced gastric cancer cell death via necroptosis. Due to the unrestricted proliferation and apoptosis resistance of PASMCs, PAH is somewhat similar to tumor disease (2). Therefore, it is highly important to inhibit the proliferation of PASMC to prevent the progression of PAH. In line with the research of Kurosawa et al. (22), we demonstrated that both low and high concentrations of celastrol significantly inhibited the proliferation of HPASMCs under hypoxic conditions by using EdU proliferation assays. To our surprise, the CCK8 assay showed that low concentrations of celastrol tended to promote the viability of HPASMCs under hypoxia. Der Sarkissian et al. (38) had previously shown that celastrol could confer cardio-protection against ischemic injury in an animal model of MI in vivo and improve cell viability under hypoxic conditions in vitro via reactive oxygen species (ROS) signaling. Additionally, considering that the optical density value at a wavelength of $450 \mathrm{~nm}$ of the CCK-8 kit depends on the cell mitochondria, we speculated that a low concentration of celastrol can protect HPASMCs from oxidative stress under hypoxia without an adequate ability 
to inhibit their proliferation. Thus, the precise effect of different concentrations of celastrol on HPASMCs and its mechanism deserve further study.

The safety of drugs is an important indicator of future clinical use. Although we did not explore the safety of celastrol in vivo, Zhao and Gong et al. $(39,40)$ had already proven that small doses of celastrol were safe in mice and rats. Combined with our finding that a low concentration of celastrol possibly promoted the viability of HPASMCs under hypoxic conditions, celastrol could one day be used to treat patients with $\mathrm{PAH}$ safely.

In conclusion, we observed favorable changes in pulmonary vascular remodeling, RV systolic pressure, RV hypertrophy, and RV function in celastrol treated rats in a model of PAH induced by MCT. In vitro, celastrol inhibited HPASMCs proliferation under hypoxia. Our results confirm that celastrol is a promising compound for the treatment of inflammation and provide relevant insights into the usage of celastrol as a future drug for PAH.

\section{Acknowledgments}

We would like to thank the American Journal Experts (AJE) for their help in polishing our paper.

Funding: This work was supported by the National Natural Science Foundation of China (grant Nos. 81770319 and 81900294).

\section{Footnote}

Reporting Checklist: The authors have completed the ARRIVE reporting checklist. Available at https://cdt. amegroups.com/article/view/10.21037/cdt-21-360/rc

Data Sharing Statement: Available at https://cdt.amegroups. com/article/view/10.21037/cdt-21-360/dss

Peer Review File: Available at https://cdt.amegroups.com/ article/view/10.21037/cdt-21-360/prf

Conflicts of Interest: All authors have completed the ICMJE uniform disclosure form (available at https://cdt.amegroups. com/article/view/10.21037/cdt-21-360/coif). The authors have no conflicts of interest to declare.

Ethical Statement: The authors are accountable for all aspects of the work in ensuring that questions related to the accuracy or integrity of any part of the work are appropriately investigated and resolved. Experiments were performed under a project license (Nos. 2020000093) granted by the Institutional Animal Use and Care Committee of Sun Yat-sen University, Guangzhou City, Guangdong Province, China, in compliance with the national or institutional guidelines for the care and use of animals. A protocol was prepared before the study without registration.

Open Access Statement: This is an Open Access article distributed in accordance with the Creative Commons Attribution-NonCommercial-NoDerivs 4.0 International License (CC BY-NC-ND 4.0), which permits the noncommercial replication and distribution of the article with the strict proviso that no changes or edits are made and the original work is properly cited (including links to both the formal publication through the relevant DOI and the license). See: https://creativecommons.org/licenses/by-nc-nd/4.0/.

\section{References}

1. Lau EMT, Giannoulatou E, Celermajer DS, et al. Epidemiology and treatment of pulmonary arterial hypertension. Nat Rev Cardiol 2017;14:603-14.

2. Thenappan T, Ormiston ML, Ryan JJ, et al. Pulmonary arterial hypertension: pathogenesis and clinical management. BMJ 2018;360:55492.

3. D'Alonzo GE, Barst RJ, Ayres SM, et al. Survival in patients with primary pulmonary hypertension. Results from a national prospective registry. Ann Intern Med 1991;115:343-9.

4. Farber HW, Miller DP, Poms AD, et al. Five-Year outcomes of patients enrolled in the REVEAL Registry. Chest 2015;148:1043-54.

5. Michelakis ED. Pulmonary arterial hypertension: yesterday, today, tomorrow. Circ Res 2014;115:109-14.

6. Tuder RM, Groves B, Badesch DB, et al. Exuberant endothelial cell growth and elements of inflammation are present in plexiform lesions of pulmonary hypertension. Am J Pathol 1994;144:275-85.

7. Mamazhakypov A, Viswanathan G, Lawrie A, et al. The role of chemokines and chemokine receptors in pulmonary arterial hypertension. Br J Pharmacol 2021;178:72-89.

8. Huertas A, Tu L, Humbert M, et al. Chronic inflammation within the vascular wall in pulmonary arterial hypertension: more than a spectator. Cardiovasc Res 2020;116:885-93.

9. Farkas D, Alhussaini AA, Kraskauskas D, et al. Nuclear factor $\mathrm{\kappa B}$ inhibition reduces lung vascular lumen 
obliteration in severe pulmonary hypertension in rats. Am J Respir Cell Mol Biol 2014;51:413-25.

10. Kannaiyan R, Shanmugam MK, Sethi G. Molecular targets of celastrol derived from Thunder of God Vine: potential role in the treatment of inflammatory disorders and cancer. Cancer Lett 2011;303:9-20.

11. Venkatesha SH, Dudics S, Astry B, et al. Control of autoimmune inflammation by celastrol, a natural triterpenoid. Pathog Dis 2016;74:ftw059.

12. Han LP, Li CJ, Sun B, et al. Protective Effects of Celastrol on Diabetic Liver Injury via TLR4/MyD88/NF- $\kappa$ B Signaling Pathway in Type 2 Diabetic Rats. J Diabetes Res 2016;2016:2641248.

13. Jaquet V, Marcoux J, Forest E, et al. NADPH oxidase (NOX) isoforms are inhibited by celastrol with a dual mode of action. Br J Pharmacol 2011;164:507-20.

14. Li Y, He D, Zhang X, et al. Protective effect of celastrol in rat cerebral ischemia model: down-regulating p-JNK, p-cJun and NF-кB. Brain Res 2012;1464:8-13.

15. Song X, Zhang Y, Dai E, et al. Mechanism of action of celastrol against rheumatoid arthritis: A network pharmacology analysis. Int Immunopharmacol 2019;74:105725.

16. Hu M, Luo Q, Alitongbieke G, et al. Celastrol-Induced Nur77 Interaction with TRAF2 Alleviates Inflammation by Promoting Mitochondrial Ubiquitination and Autophagy. Mol Cell 2017;66:141-153.e6.

17. Cheng M, Wu G, Song Y, et al. Celastrol-Induced Suppression of the MiR-21/ERK Signalling Pathway Attenuates Cardiac Fibrosis and Dysfunction. Cell Physiol Biochem 2016;38:1928-38.

18. Ye S, Luo W, Khan ZA, et al. Celastrol Attenuates Angiotensin II-Induced Cardiac Remodeling by Targeting STAT3. Circ Res 2020;126:1007-23.

19. Calvier L, Chouvarine P, Legchenko E, et al. PPAR $\gamma$ Links BMP2 and TGF $\beta 1$ Pathways in Vascular Smooth Muscle Cells, Regulating Cell Proliferation and Glucose Metabolism. Cell Metab 2017;25:1118-1134.e7.

20. Dawson S, Lawrie A. From bones to blood pressure, developing novel biologic approaches targeting the osteoprotegein pathway for pulmonary vascular disease. Pharmacol Ther 2017;169:78-82.

21. Nogueira-Ferreira R, Vitorino R, Ferreira R, et al. Exploring the monocrotaline animal model for the study of pulmonary arterial hypertension: A network approach. Pulm Pharmacol Ther 2015;35:8-16.

22. Kurosawa R, Satoh K, Nakata T, et al. Identification of Celastrol as a Novel Therapeutic Agent for Pulmonary
Arterial Hypertension and Right Ventricular Failure Through Suppression of Bsg (Basigin)/CyPA (Cyclophilin A). Arterioscler Thromb Vasc Biol 2021;41:1205-17.

23. Hassoun PM, Mouthon L, Barberà JA, et al. Inflammation, growth factors, and pulmonary vascular remodeling. J Am Coll Cardiol 2009;54:S10-9.

24. Rabinovitch M, Guignabert C, Humbert M, et al. Inflammation and immunity in the pathogenesis of pulmonary arterial hypertension. Circ Res 2014;115:165-75.

25. Abid S, Marcos E, Parpaleix A, et al. CCR2/CCR5mediated macrophage-smooth muscle cell crosstalk in pulmonary hypertension. Eur Respir J 2019;54:1802308.

26. Cascão R, Vidal B, Lopes IP, et al. Decrease of CD68 Synovial Macrophages in Celastrol Treated Arthritic Rats. PLoS One 2015;10:e0142448.

27. Hu Y, Chi L, Kuebler WM, et al. Perivascular Inflammation in Pulmonary Arterial Hypertension. Cells 2020;9:2338.

28. Hashimoto-Kataoka T, Hosen N, Sonobe T, et al. Interleukin-6/interleukin-21 signaling axis is critical in the pathogenesis of pulmonary arterial hypertension. Proc Natl Acad Sci U S A 2015;112:E2677-86.

29. Karin M. Nuclear factor-kappaB in cancer development and progression. Nature 2006;441:431-6.

30. Sethi G, Tergaonkar V. Potential pharmacological control of the NF- $\kappa$ B pathway. Trends Pharmacol Sci 2009;30:313-21.

31. Lawrence T. The nuclear factor NF-kappaB pathway in inflammation. Cold Spring Harb Perspect Biol 2009;1:a001651.

32. Sethi G, Ahn KS, Pandey MK, et al. Celastrol, a novel triterpene, potentiates TNF-induced apoptosis and suppresses invasion of tumor cells by inhibiting NFkappaB-regulated gene products and TAK1-mediated NFkappaB activation. Blood 2007;109:2727-35.

33. Wang XN, Wu Q, Yang X, et al. Effects of Celastrol on growth inhibition of U937 leukemia cells through the regulation of the Notch1/NF-kappaB signaling pathway in vitro. Chin J Cancer 2010;29:385-90.

34. DiDonato JA, Mercurio F, Karin M. NF- $\kappa$ B and the link between inflammation and cancer. Immunol Rev 2012;246:379-400.

35. Sethi G, Sung B, Aggarwal BB. Nuclear factor-kappaB activation: from bench to bedside. Exp Biol Med (Maywood) 2008;233:21-31.

36. Corson TW, Crews CM. Molecular understanding and modern application of traditional medicines: triumphs and 
trials. Cell 2007;130:769-74.

37. Guo D, Zhang W, Yang H, et al. Celastrol Induces Necroptosis and Ameliorates Inflammation via Targeting Biglycan in Human Gastric Carcinoma. Int J Mol Sci 2019;20:5716.

38. Der Sarkissian S, Cailhier JF, Borie M, et al. Celastrol protects ischaemic myocardium through a heat shock response with up-regulation of haeme oxygenase-1. Br J Pharmacol 2014;171:5265-79.

39. Zhao J, Luo D, Zhang Z, et al. Celastrol-loaded PEG-

Cite this article as: Li H, Liu Q, Yue Y, Wang S, Huang S, Huang L, Luo L, Zhang Y, Wu Z. Celastrol attenuates the remodeling of pulmonary vascular and right ventricular in monocrotaline-induced pulmonary arterial hypertension in rats. Cardiovasc Diagn Ther 2022;12(1):88-102. doi: 10.21037/cdt21-360
PCL nanomicelles ameliorate inflammation, lipid accumulation, insulin resistance and gastrointestinal injury in diet-induced obese mice. J Control Release 2019;310:188-97.

40. Gong T, Zhang P, Deng C, et al. An effective and safe treatment strategy for rheumatoid arthritis based on human serum albumin and Kolliphor® HS 15. Nanomedicine (Lond) 2019;14:2169-87. 